\title{
A Study of Recreation in the Renewal of Historical Blocks
}

\author{
Ma Mingchun \\ School of Civil Engineering and Architecture \\ University of Jinan \\ Jinan, China \\ mamingchun@163.com
}

\author{
Liu Qiang \\ School of Civil Engineering and Architecture \\ University of Jinan \\ Jinan, China
}

\begin{abstract}
The purpose of this essay is to reshape urban vitality and to study three kinds of material space of historical blocks, mainly including residential buildings, street space and open node renewal under the guidance of national policies of "city double repair". On the basis of research and analysis of my case study of Jinan Wang Fu Pond block, the specific principle and implementation methods which consist of the updating of residential building based on upgrading the living environment, the updating of street space based on the space series, the updating of open node based on the vitality analysis will be put forward in my essay.
\end{abstract}

Keywords-historical block, vitality remold, open node renewal, urban renewal

\section{INTRODUCTION}

In March 6, 2017, the Ministry of housing and Urban Construction issued the document which is "on the strengthening of the ecological restoration, guidance on city repair work" to arrange to carry out a comprehensive ecological restoration, repair and repair work of city, city goals in addition to improving the old urban living environment, the traditional plastic block vitality is its core content. Therefore, at this stage of history block update, should be combined with the block vitality plastic renewal and physical space, this article will focus on the block vitality plasticity update specific implementation way in the process of physical space.

Jinan is a national historical and cultural city, the palace pond block was designated in 2014 as a provincial historical and cultural blocks within the scope, basically a continuation and retain the integrity of the traditional scale pattern and block style. So the renewal of more representative and promote the significance.(Fig1)

This work was financially supported by the Project of Humanities and Social Science in Universities in Shandong(SQ16021), the Natural Science Foundation of University of Jinan(xky1617), the Key Subject of Art and Science in Shandong Province 1607247.

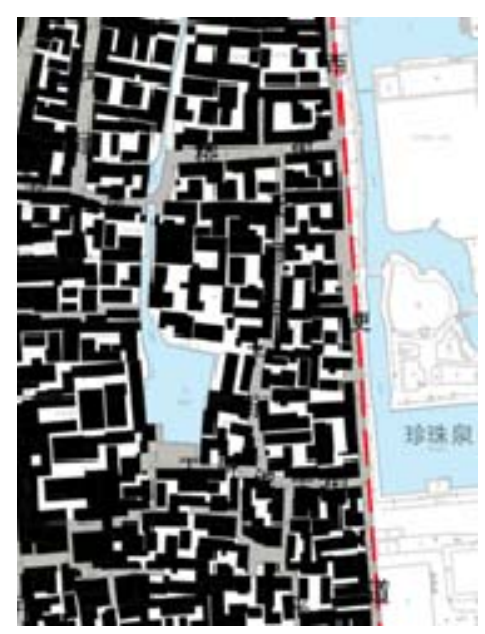

Fig. 1. The Texture of WangFu pond

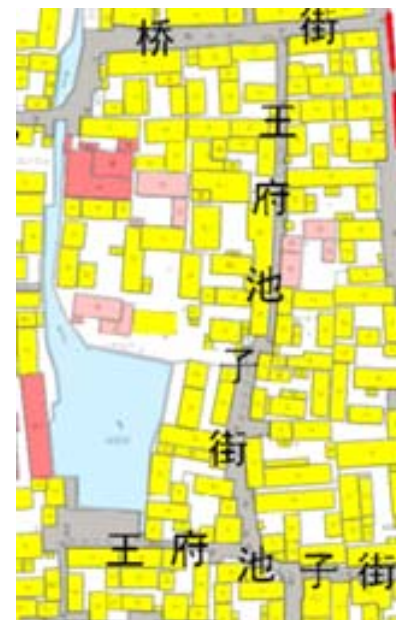

Fig. 2. The distribution diagram of building function

\section{General Situation of WANG Fu Pond Block}

According to the survey, the Wang Fu Pond Street covers an area of about one hectare, with the north-south and east-west WangFu Pond street road skeleton. The buildings were built in the last century, around $80 \mathrm{~s}$, basically intact, a part of them was built in the last century 20s. Mainly residential land, accounting for $60 \%$ of the total land. A small amount of water culture theme of family hotels and restaurants covers $10 \%$ of the total 
land. In addition, the public open space covers $30 \%$ in Wang $\mathrm{Fu}$ Pond block. Therefore, this area belongs to the history of residential neighborhoods, as living function, also with Zhuo ying Spring waters as the main open space. (Fig2)

\section{The MaIn Way to Realize the Renewal of Block Vitality IN THE PROCESS OF MATERIAL SpaCe RENEWAL}

Residential neighborhoods have the characteristics of historical continuity, integrity and of the space community. The neighborhood residents' living habits, neighborhood relations and cultural intangible cultural aspects, are to be considered the dynamic plasticity of Resident Historic District. In the survey, the area of residential building is damaged seriously and lack of constructions. The Zhuoying Spring water is near the open space and street corridor Wang Fu Pond node is considered to be less. Therefore, according to the actual situation of the land, how to put forward dynamic plasticity is mainly through the following ways:

\section{A. Based on improving the living environment of residential building protection and updating}

The historic buildings in the classification of the existing protection updates, higher value of the dwelling for full protection, and for the existing poor quality. The high value residential buildings can be appropriate to reform, improve the living environment. The original residents -- the main elements retain its vigor. The relationship of the original social network maintains the authenticity protection. The lives of residents can enhance the diversity of city life and bring more vitality. (Fig3,Fig4)

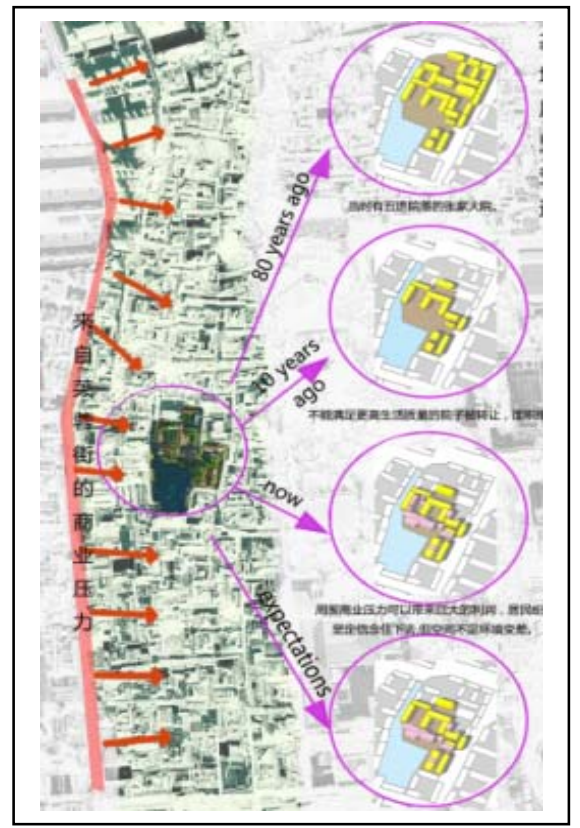

Fig. 3. Transition analysis diagram of Zhang courtyard

The most typical residential neighborhood pond palace Group - Zhang's family courtyard to conduct in-depth research, found that the current problems and puts forward the corresponding countermeasures. The Zhang's family courtyard lived seventeenth generation 22 generation grandson, a total of
12 households in 5 generations living here and live together in seven yards. Three of them in the courtyard is good quality in construction, can fully protect the houses, while the other four buildings in the courtyard are poor quality and low value. Recommendations can be reconstructed in the residential building blocks keep overall coordination principle, can increase the amount of commercial and hotel function, enhance the diversity of function blocks. (Fig5)

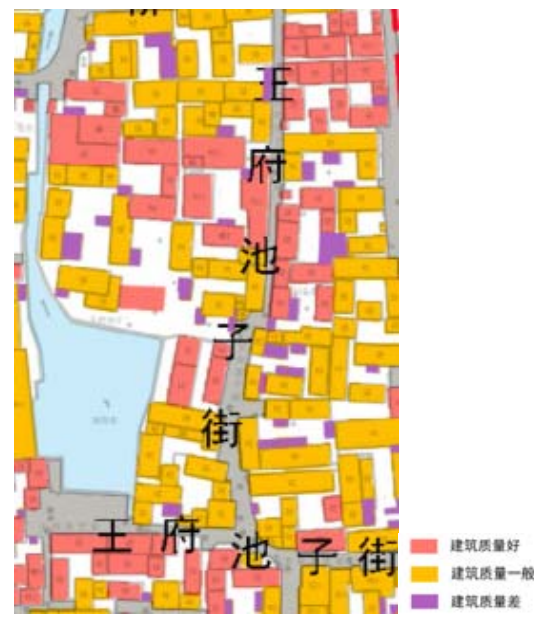

Fig. 4. The layout plan of construction quality

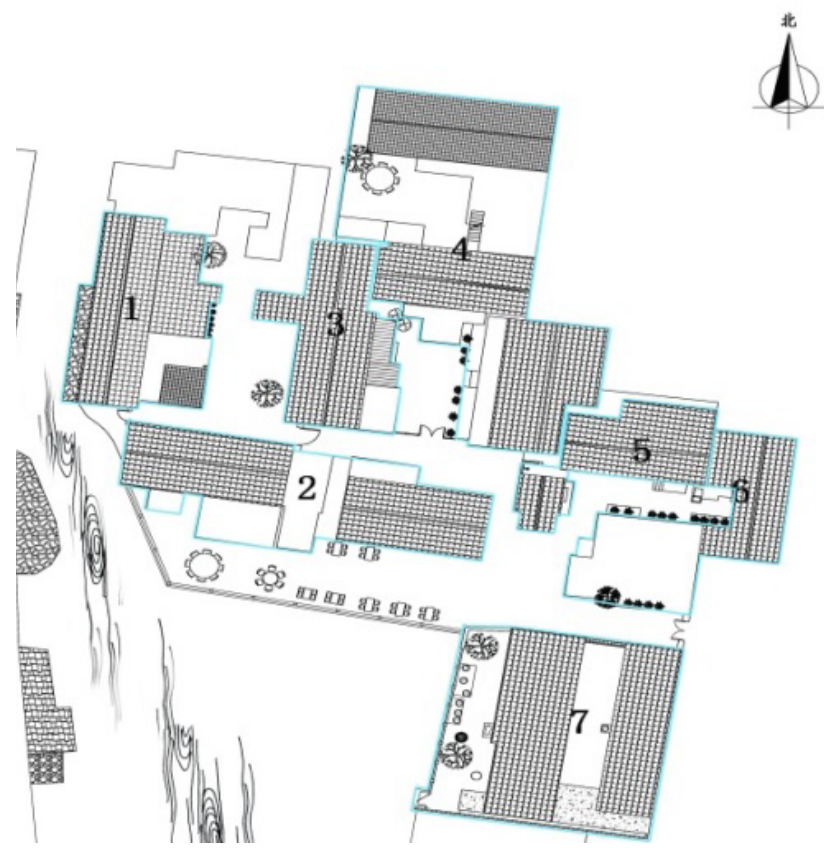

Fig. 5. The layout of Zhang's Family

\section{B. The renewal of street space based on spatial sequence}

The British city design theory leader Gordon Cullen think the street landscape is not stable tableaux continuous system but spatial awareness, people's feelings from experience to experience things and hope. The influence of the contemporary American landscape architect John.O.Simonds also made a 
similar point of view. People from the movement which can obtain a series of the continuity of the perception formed the so-called sequence, sequence significance lies in people's perception and experience. People tend to feel that a dynamic, because the sequence can generate movements and trends suggest that the beginning of the end of the series to exercise

The Wang Fu Pond block is located in the historical and cultural blocks within the scope of protection in Jinan city by the East-West and North-South Street. Wang Fu Pond Street has a length of one hundred meters, with a width of $2.5-3.5$ meters. The length of Wang Fu Pond Street's 60 meters and 23 meters wide. The street space show strong linear guide, space scale, and rich space perception (Fig6,Fig7)

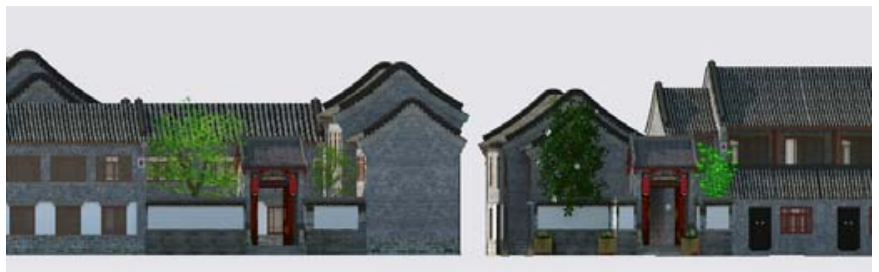

Fig. 6. The street façade updates 1

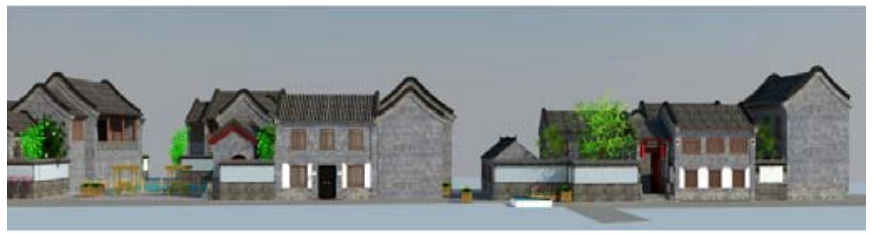

Fig. 7. The street façade update 2

Therefore, the street space considers the user experience, and controls the space sequence such as scale, shape, color, texture and other design which can be stimulated, resulting in a rhythmic rhythm. Especially for the formation of key road, the scene focuses on fully considerations. Open node updating based on the vitality analysis

The vitality analysis is one of the theories of generalized behavior analysis, with the help of sociology and psychology, the analysis of space in history, culture, human activities and other factors are rich. It pays attention to the "people" activities, creating diverse activities. The use of functional space, meeting the various needs of users, is to keep the city vitality and open. The open node in the block is the most intensive, concentrated, and popular space. (Fig8)

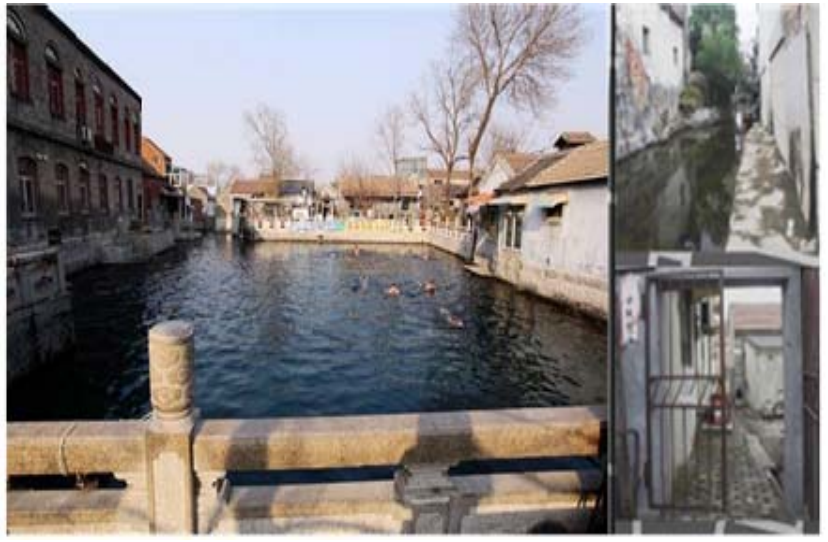

Fig. 8. The block environment of Wang Fu Pond

Wang Fu Pond (Zhuoying Spring) is the only one node to be open, more than thirty meters long from north to south, east 30 meters, water area of 600 square meters, adjacent to the east of Zhang family courtyard dwellings Hospital No. 7, north three meters apart is the Zhang family courtyard hospital No. 4, along the west side is a city of about twenty meters long two the hotel building, on the south side there is a small open square depth of about five meters. The spring flows from the northwest side, basically in the household to flow through, Qushuiting Street, and then into the Da Ming Lake Jinan Springs Scenic Area. And the most unique folk activities throughout the year of Wang Fu pond is swimming. The swimming especially almost becomes the old city scene. In addition, sitting by the water, drinking a bowl of tea, pulling the home life, are the pleasure and entertainment of the old people.

Therefore, in order to maintain the vitality of the node space, it should be on the perspective of the need to make corresponding design on the surrounding environment. On the north side of concession space renovation and landscaping, it shall enhance the quality of open space and form the waterfront viewing platform. The south side of the square is connected with the east side of the platform and increases the tour route, also rich viewing angle as well. (Fig9,Fig10)

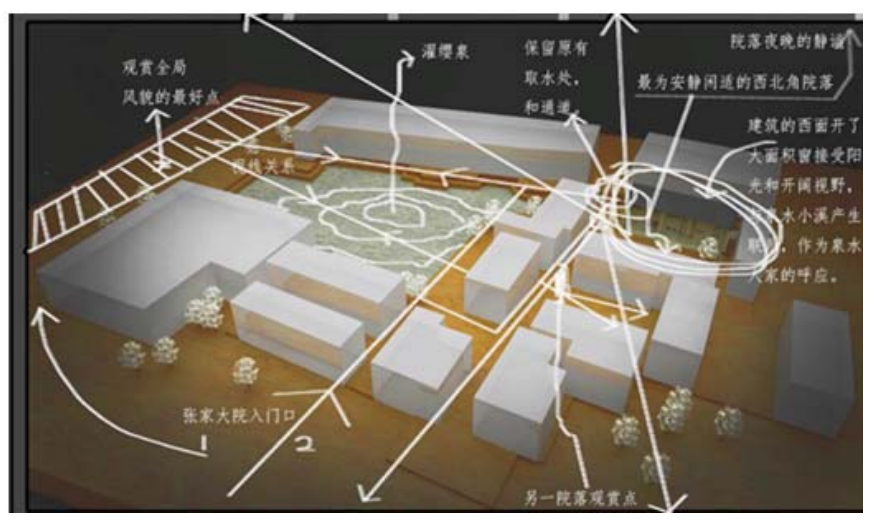

Fig. 9. The main sight relations of Wang Fu Pond and surroundings. 


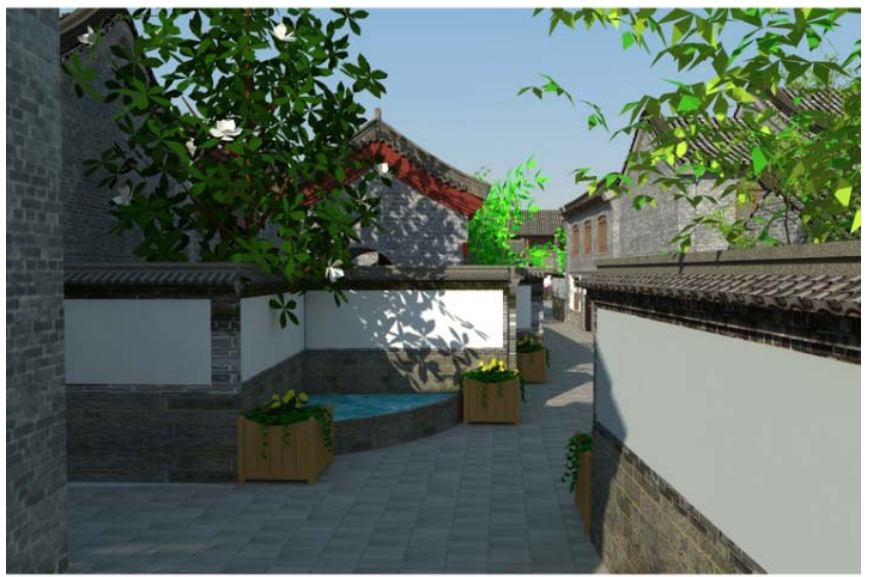

Fig. 10. The update of street node

\section{SUMMARY}

Jacobs proposes to recover the neighborhood "diversity" vitality and the city design must meet four basic conditions: land, mixed function of short streets, old buildings, a certain population density. These four conditions are present in the historic district, in the national policy of "city double repair" under the guidance of the historical block in spring. As long as we take the energy remold as a target, and a more characteristic and viable old city would be surely created.

\section{ACKNOWLEDGEMENTS}

This work was financially supported by the Project of Humanities and Social Science in Universities in Shandong(SQ16021), the Natural Science Foundation of University of Jinan (xky1617), the Key Subject of Art and Science in Shandong Province 1607247.

\section{REFERENCES}

[1] G.Gullen,Townscape.New York:Reinhold Publishing Corporation,1961.

[2] Jane.Jacobs, The Death and Life of Great American Cities,Nanjing,2007

[3] Zhang Jianhua,Mamingchun, Thoughts About Experience Tourism and Jinan's Urban Landscape Shaping as A historical and Cultural City,Urban Studies,2008(3)

[4] Wei Xiangdong \&Song Yanqi.(2005) Urban Landscape. Beijing: China Forestry Press.

[5] Wang Jianguo.(2005) Urban Design. Nanjing: Southeast University Press.

[6] Liu Binyi \& Liu Qin. (2006) Urban Landscape Planning Which Serves City Tourist Image-A Case Study in Nanjing City. 03 